MATHEMATICS OF COMPUTATION

Volume 72, Number 242, Pages 1051-1054

S 0025-5718(02)01445-X

Article electronically published on June 6, 2002

\title{
THE SMALLEST SOLUTIONS TO THE DIOPHANTINE EQUATION
}

$$
x^{6}+y^{6}=a^{6}+b^{6}+c^{6}+d^{6}+e^{6}
$$

GIOVANNI RESTA AND JEAN-CHARLES MEYRIGNAC

\begin{abstract}
In this paper we discuss a method used to find the smallest nontrivial positive integer solutions to $a_{1}^{6}+a_{2}^{6}=b_{1}^{6}+b_{2}^{6}+b_{3}^{6}+b_{4}^{6}+b_{5}^{6}$. The method, which is an improvement over a simple brute force approach, can be applied to search the solution to similar equations involving sixth, eighth and tenth powers.
\end{abstract}

\section{INTRODUCTION}

Diophantine equations of the form $\sum_{i=1}^{m} a^{s}=\sum_{j=1}^{n} b^{s}$ have attracted interest since antiquity, and solutions for various values of $s, m$ and $n$ are known. The most comprehensive report to date is 1], while the first systematic computer search is described in [2]. In this paper we determine the first nontrivial solutions to the equation

$$
a_{1}^{6}+a_{2}^{6}=b_{1}^{6}+b_{2}^{6}+b_{3}^{6}+b_{4}^{6}+b_{5}^{6} .
$$

The first 5 primitive solutions, i.e., with $\operatorname{gcd}\left(a_{1}, a_{2}, b_{1}, \ldots, b_{5}\right)=1$, are

$$
\begin{aligned}
& 1117^{6}+770^{6}=1092^{6}+861^{6}+602^{6}+212^{6}+84^{6} \\
& 2041^{6}+691^{6}=1893^{6}+1468^{6}+1407^{6}+1302^{6}+1246^{6} \\
& 2441^{6}+752^{6}=2184^{6}+2096^{6}+1484^{6}+1266^{6}+1239^{6} \\
& 2827^{6}+151^{6}=2653^{6}+2296^{6}+1488^{6}+1281^{6}+390^{6} \\
& 2959^{6}+2470^{6}=2954^{6}+2481^{6}+850^{6}+798^{6}+420^{6} .
\end{aligned}
$$

In the following we discuss the method used to find the solutions, which is based on a brute force decomposition algorithm made feasible by restricting the trials by means of modular arithmetic considerations.

\section{THE METHOD}

Since $x^{6} \equiv 0(\bmod 7)\left(\right.$ resp., $\left.x^{6} \equiv 1(\bmod 7)\right)$ when 7 divides (resp., does not divide) $x$, for any $i=1,2, \ldots, 6$ we have $x_{1}^{6}+\cdots+x_{i}^{6} \equiv n_{x}(\bmod 7)$, where $n_{x}$ denotes the number of $x_{i}$ which are not divisible by 7 .

Thus in any primitive solution to (1) we have either $n_{a}=n_{b}=1$ or $n_{a}=n_{b}=2$.

Received by the editor May 24, 1999 and, in revised form, April 3, 2001 and July 9, 2001. 2000 Mathematics Subject Classification. Primary 11D41, 11Y50.

Key words and phrases. Diophantine equations, computational number theory.

(C)2002 American Mathematical Society 
Either way, we can assume, reordering the $b_{i}$ 's if necessary, that 7 divides $b_{3}, b_{4}$ and $b_{5}$. Hence we have

$$
b_{2}^{6} \equiv a_{1}^{6}+a_{2}^{6}-b_{1}^{6} \quad\left(\bmod 7^{6}\right) .
$$

Since we are looking for primitive solutions, we have assumed that $n_{a}=n_{b}>0$. Hence at least one of $b_{1}$ and $b_{2}$ must be not divisible by 7 . Thus we can assume that $b_{2}$ (and hence $a_{1}^{6}+a_{2}^{6}-b_{1}^{6}$ ) is not divisible by 7 .

Our method, which is inspired by the one used in [2] to search (without success) for solutions to $a_{1}^{6}=b_{1}^{6}+b_{2}^{6}+b_{3}^{6}+b_{4}^{6}+b_{5}^{6}+b_{6}^{6}$, is based on the above considerations, and proceeds as follows.

For every $a_{1}, a_{2} \leq a_{1}$ and $b_{1}$ such that $a_{1}^{6}+a_{2}^{6} \geq b_{1}^{6}$ and $a_{1}^{6}+a_{2}^{6}-b_{1}^{6} \not \equiv 0\left(\bmod 7^{6}\right)$, we solve the congruence

$$
x^{6} \equiv a_{1}^{6}+a_{2}^{6}-b_{1}^{6} \quad\left(\bmod 7^{6}\right)
$$

with respect to $x$, obtaining a suitable set $B$ of possible values for $b_{2}$.

The modular equation $x^{6} \equiv t\left(\bmod 7^{6}\right)$ has exactly 6 solutions below $7^{6}$ when $t \equiv 1(\bmod 7)$, and no solutions at all for other values of $t$ which are non-zero $\bmod$ 7. Hence the set $B$ has a cardinality that does not exceed $6\left\lceil\left(a_{1}+a_{2}\right) / 7^{6}\right\rceil$, and can be easily determined, once the solutions to $x^{6} \equiv t\left(\bmod 7^{6}\right)$ for $0<t<7^{6}$ have been precomputed.

For every value $b_{2} \in B$ such that $v=a_{1}^{6}+a_{2}^{6}-\left(b_{1}^{6}+b_{2}^{6}\right)$ is positive, we try to decompose $v / 7^{6}$ as $v / 7^{6}=c_{1}^{6}+c_{2}^{6}+c_{3}^{6}$. If such $c_{j}$ 's do exist, we have obtained a solution to (1), where $b_{3}=7 c_{1}, b_{4}=7 c_{2}$, and $b_{5}=7 c_{3}$.

This method, despite its simplicity, is faster than the naïve brute force approach based on the trial decomposition of $a_{1}^{6}+a_{2}^{6}$ as a sum of five sixth powers. This depends mainly on two factors: a) the few possible values for $b_{2}$ (at most 6 in the range we have so far explored, i.e., $\left.a_{1}, a_{2} \leq 30,400\right)$, and b) the trial decomposition of $v / 7^{6}$ instead of $v$.

The method can be further refined by noticing that the property of the sixth powers that we have described for modulus 7 also holds with respect to moduli 8 and 9 . In other words, $x^{6}(\bmod 8)$ is 0 for $x$ even and 1 for $x$ odd, and analogously $x^{6}(\bmod 9)$ is 0 for $x$ a multiple of 3 and 1 otherwise.

The first immediate consequence is that we can discard all the configurations such that $a_{1}$ and $a_{2}$ are both multiples of 2 or 3 , since we cannot obtain a primitive solution. More generally, we can exploit the following two observations to reduce the efforts needed to decompose a number $y$ into the sum $x_{1}^{6}+\cdots+x_{k}^{6}$ for $k<7$.

- If $y \equiv 0(\bmod 7)((\bmod 8)$ or $(\bmod 9)$, respectively), then 7 (2 or 3 , respectively) must divide all the $x_{i}$ 's. In turn this implies that the problem can be reduced to the decomposition of $y / 7^{6}$ (respectively $y / 2^{6}$ or $y / 3^{6}$ ) if such a number is an integer. If not, the decomposition does not exist.

- If $y \equiv 1(\bmod 7)((\bmod 8)$ or $(\bmod 9)$, respectively), then we can assume that one of the $x_{i}$ 's, say $x_{1}$, satisfies $y^{6} \equiv x_{1}^{6}\left(\bmod 7^{6}\right)\left(\left(\bmod 2^{6}\right)\right.$ or $(\bmod$ $\left.3^{6}\right)$, respectively) and we can restrict the search space accordingly.

Moreover, all the values $x^{6}>0$ can be expressed as $(8 k+1) 2^{n}$ for suitable values of $k$ and $n$. This implies that $x^{6}+y^{6}$, for every $x, y>0$, is of the form $(4 j+1) 2^{m}$. Therefore it is possible to devise a simple criterion, based on the binary decomposition of a number $t$, to discard several values of $t$ that cannot be expressed as $x^{6}+y^{6}$. 
Finally we observe that straightforward modular considerations allow us to further restrict the search space. For example, we note that the sum of 3 sixth powers cannot be equal to 4 or $9(\bmod 13)$, nor equal to 5,16 or $17(\bmod 19)$. Similar constraints, with respect to moduli $13,19,31,37,43,61,67,73,79,109,121,139$, 223 and 529 , have been used to discard illegal values during the decomposition.

\section{IMPLEMENTATION}

The method was first implemented in $\mathrm{C}$, using a public domain package for multiple precision arithmetic and a custom 91-bits integer representation for the trial decomposition. The first version of the program did not use the technique explained above to speed up the trial decomposition of $a_{1}^{6}+a_{2}^{6}-b_{1}^{6}$.

The program used about $20 \mathrm{Mb}$ of memory, essentially to maintain a sorted array of all the values $x^{6}+y^{6}$ under a suitable limit to improve the performance of the trial decomposition.

The program ran on a $\mathrm{PC}$ with a Pentium2 $\mathrm{CPU}$ at $400 \mathrm{MHz}$ and $256 \mathrm{Mb}$ of central memory, under Windows NT, and found the smallest solution in about 5 minutes 11

TABLE 1.

\begin{tabular}{|ll|lllll|}
\hline$a_{1}$ & $a_{2}$ & $b_{1}$ & $b_{2}$ & $b_{3}$ & $b_{3}$ & $b_{5}$ \\
\hline 1117 & 770 & 1092 & 861 & 602 & 212 & 84 \\
2041 & 691 & 1893 & 1468 & 1407 & 1302 & 1246 \\
2441 & 752 & 2184 & 2096 & 1484 & 1266 & 1239 \\
2827 & 151 & 2653 & 2296 & 1488 & 1281 & 390 \\
2959 & 2470 & 2954 & 2481 & 850 & 798 & 420 \\
6623 & 323 & 6615 & 2912 & 642 & 434 & 363 \\
8905 & 347 & 8820 & 5489 & 2576 & 2499 & 534 \\
8969 & 5203 & 9023 & 2604 & 2520 & 2379 & 478 \\
9551 & 9451 & 10080 & 8589 & 4884 & 3976 & 3943 \\
9612 & 7271 & 9198 & 7446 & 6580 & 6279 & 5118 \\
9707 & 6277 & 9675 & 5796 & 5531 & 4536 & 3640 \\
12272 & 2459 & 11522 & 9144 & 8283 & 7434 & 1400 \\
13417 & 5933 & 11403 & 11378 & 10698 & 4641 & 70 \\
13903 & 5317 & 13788 & 8295 & 6026 & 1827 & 1232 \\
15149 & 6914 & 14520 & 10675 & 10444 & 6006 & 810 \\
15627 & 1865 & 14196 & 13608 & 5385 & 4032 & 565 \\
15905 & 10519 & 14679 & 13759 & 9534 & 3822 & 2482 \\
16103 & 9111 & 14868 & 13755 & 8484 & 6253 & 2004 \\
16159 & 10532 & 15442 & 11760 & 11016 & 10273 & 3234 \\
16160 & 1865 & 15792 & 11470 & 4984 & 4329 & 1008 \\
16315 & 8749 & 15246 & 11682 & 11375 & 11140 & 7119 \\
16867 & 14786 & 17790 & 9821 & 9786 & 2912 & 2502 \\
17737 & 16043 & 17646 & 14574 & 14261 & 3255 & 2590 \\
19168 & 1747 & 18984 & 10584 & 10088 & 8214 & 5299 \\
19869 & 10673 & 19866 & 9783 & 9324 & 2088 & 1925 \\
\hline
\end{tabular}

1 The smallest solution to the equation under investigation here has also been discovered, independently, by E. Brisse while he participated in the project [4]. 
Subsequently, we implemented a version which takes into account all the techniques illustrated in the previous section, and which is written in assembly language to further improve performance.

Table 1 summarizes the primitive solutions in the range $a_{1}, a_{2}<20,000$. The 5 smallest were found by means of the first implementation of the method, and the others were found by the computational efforts of the participants in the project [4], to whom we have granted access to the improved version of our algorithm.

\section{FURTHER WORK}

So far, we have computed all solutions with $a_{1}, a_{2} \leq 30,400$. In particular, no solution to the related equation $a_{1}^{6}+a_{2}^{6}=b_{1}^{6}+b_{2}^{6}+b_{3}^{6}+b_{4}^{6}$ exists below 30,400. The similar equation $a_{1}^{6}=b_{1}^{6}+b_{2}^{6}+b_{3}^{6}+b_{4}^{6}+b_{5}^{6}+b_{6}^{6}$ is the subject of a current distributed search [4]. So far no solution has been found for $a_{1}<730,000$.

Following the heuristic arguments of [3], one might expect that there are $O(x)$ solutions to (1), as the variables run in the range $0, \ldots, x$. Indeed there are $O\left(x^{7}\right)$ choices for the seven variables, while $\left|a_{1}^{6}+a_{2}^{6}-b_{1}^{6}-b_{2}^{6}-b_{3}^{6}-b_{4}^{6}-b_{5}^{6}\right|$ lies in the range $0, \ldots, 5 x^{6}$. Assuming, quite forcefully, that each number in this range is taken with equal probability, then we expect that the number of solutions is proportional to $x$. Experimental results support this line of reasoning, since the total number of solutions with the variables in the ranges $0, \ldots, 10,000,10,000, \ldots, 20,000$ and $20,000, \ldots, 30,000$ is 27,46 and 52 , respectively, or 10,15 , and 18 , counting only the primitive solutions.

Some algorithms based on the same ideas exploited in this paper are being used to search for solutions to similar equations involving sums of eighth and tenth powers. As an example, for eighth powers, we use reductions with respect to 256 and $5^{8}$ instead of 8 and $7^{6}$, and modular constraints 17, 841 and 1681. For tenth powers, we use $2^{10}$ and $11^{5}$ and moduli 25, 31, 41, and 61 . Several results concerning eighth and tenth powers have been obtained, and an updated list of solutions is maintained in 4 .

Scott I. Chase independently searched 8 th, 10th, 12th and 16th powers with a similar method, and found several solutions, among which the most interesting is $966^{8}+539^{8}+81^{8}=954^{8}+725^{8}+481^{8}+310^{8}+158^{8}$.

\section{ACKNOWLEDGMENTS}

We wish to thank an anonymous referee for pointing out reference [3], and suggesting some extensions to our results.

\section{REFERENCES}

1. R.L. Ekl, New Results in Equal Sums of Like Powers. Math. Comp. 67 (1998), 1309-1315. MR 98m:11023

2. L.J. Lander, T.R. Parkin, J.L. Selfridge, A Survey of Equal Sums of Like Powers. Math. Comp. 21 (1967), 446-459. MR 36:5060

3. B. Mazur, Questions about Powers of Numbers. Notices of the AMS, February 2000 195-202. MR 2000k:11111

4. J.-C. Meyrignac, et al., Computing Minimal Equal Sums of Like Powers. Distributed computing project, see http://euler.free.fr.

Istituto di Matematica Computazionale -CNR, Pisa, Italy.

E-mail address: resta@imc.pi.cnr.it

E-mail address: euler@free.fr 